\title{
Factors associated with the number and size of renal angiomyolipomas in sporadic angiomyolipoma (sAML): a study of adult patients with sAML managed in a Dutch tertiary referral center
}

\author{
J. L. H. Ruud Bosch ${ }^{1}$ Francis Vekeman ${ }^{2} \cdot$ Mei Sheng Duh ${ }^{3} \cdot$ Maureen Neary $^{4} \cdot$ Matthew Magestro $^{4}$. \\ Jonathan Fortier ${ }^{2} \cdot$ Paul Karner $^{3} \cdot$ Raluca lonescu-Ittu ${ }^{2} \cdot$ Bernard A. Zonnenberg $^{5}$ (i)
}

Received: 21 July 2017 / Accepted: 5 December 2017 / Published online: 15 January 2018

(C) The Author(s) 2018. This article is an open access publication

\begin{abstract}
Purpose To describe the patient characteristics, treatments, disease monitoring, and kidney function of patients with sporadic angiomyolipoma (sAML), stratified by the number and size of renal angiomyolipomas (AMLs).

Methods Single-center retrospective analysis of patients with sAML treated from 1990 to 2015 in a dedicated clinic for inheritable tumor syndromes in a tertiary referral center from the Netherlands. Patients' first AML assessment at the clinic was defined as the index date. Patient characteristics were measured at the index date. Treatments, disease monitoring, and kidney function were measured post-index date.

Results The study sample included 53 patients followed for a total of 184.6 patient-years. At the index date, the largest AML was $\geq 3.5 \mathrm{~cm}$ for 26 patients and $<3.5 \mathrm{~cm}$ for 27 patients (including six patients with five or more AMLs of $<3.5 \mathrm{~cm}$ ). As compared to patients with AMLs $<3.5 \mathrm{~cm}$, patients with largest AML $\geq 3.5 \mathrm{~cm}$ had higher frequency of pre-index bleeding episodes (31 vs. $4 \%$ ), pre-index hypertension (35 vs. 15\%), post-index nephrectomy (19 vs. $4 \%$ ), post-index embolization (8 vs. $0 \%$ ), and post-index renal scans (1.14 vs. 0.74 scans/year). Kidney impairment was especially pronounced in young adults with $A M L \geq 3.5 \mathrm{~cm}$. On average, patients with $\mathrm{sAML}$ developed chronic kidney disease stage two earlier than the general Dutch population (age 42 vs. 55 years), but later than the patients with tuberous sclerosis complex (35 years).

Conclusions Patients with sAML, especially those with larger AMLs, have high disease burden.
\end{abstract}

Keywords Angiomyolipoma $\cdot$ Treatment $\cdot$ Disease monitoring $\cdot$ Kidney function

\section{Introduction}

Electronic supplementary material The online version of this article (https://doi.org/10.1007/s11255-017-1766-9) contains supplementary material, which is available to authorized users.

Bernard A. Zonnenberg

B.Zonnenberg@kpnmail.nl

1 Department of Urology, University Medical Center Utrecht, Heidelberglaan 100, 3584 CX Utrecht, Netherlands

2 Groupe d'analyse, Ltée, 1000 De La Gauchetiere West, Suite 1200, Montréal, QC H3B 4W5, Canada

3 Analysis Group, Inc., 111 Huntington Avenue 14th Floor, Boston, MA 02199, USA

4 Novartis Pharmaceuticals Corporation, One Health Plaza, East Hanover, NJ 07936, USA

5 Department of Internal Medicine, University Medical Center Utrecht, Heidelberglaan 100, 3584 CX Utrecht, Netherlands
Renal angiomyolipoma (AML) accounts for $2-6 \%$ of all kidney tumors [1]. A retrospective study of 61,389 patients without tuberous sclerosis complex (TSC) who underwent abdominal ultrasounds during routine care found an AML prevalence of $0.60 \%$ in females and $0.28 \%$ in males [2]. AML usually develops sporadically (sAML), but in up to $20 \%$ of the cases it may be associated with genetic disorders such as TSC [3]. sAML has later age onset, higher prevalence in women, and generally grows more slowly than TSCrelated AML (TSC-AML) [4-6]. The literature on sAMLs is mostly based on small case series [6-9], and little is known about the natural history of sAML and patterns of treatment in clinical studies. Previous studies have shown that AML tumors grow at an average rate of $0.021-0.19 \mathrm{~cm} /$ year [6, 
$10,11]$, leading sometimes to life-threatening complications such as aneurysmal vessel rupture or retroperitoneal hemorrhage $[12,13]$.

Embolization and nephrectomy are interventions recommended to patients at higher risk of potentially life-threatening bleeding complications and flank pain due to large AMLs [14]. While these invasive interventions are reliable in preventing or treating active bleeding and for improving flank pain symptoms, they may affect kidney function by damaging the renal tissue [15]. Active surveillance is the most common sAML disease management option not involving an invasive kidney intervention, although mammalian target of rapamycin inhibitors (mTORi) may be used off-label in some patients with sAML, based on their mechanism of action in TSC-related AML [16].

This study describes patient characteristics, treatments, disease monitoring, and kidney function of patients with sporadic angiomyolipoma (sAML), stratified by the number and size of renal angiomyolipomas.

\section{Methods}

The study used a single-center retrospective chart-review study design. Data were collected from the medical charts of patients managed at the University Medical Center Utrecht (UMCU) from January 1990 to April 2015. The UMCU is a major specialty center in the Netherlands for patients with kidney tumors and a tertiary center with dedicated clinic for inheritable tumor syndromes. Most patients receive their first kidney tumor diagnosis at UMCU after an ultrasound or computerized tomography (CT) scan referral, but previously diagnosed patients may also be referred for second opinion or may present with acute bleeding in the emergency department.

Patients with an imaging or pathological diagnosis of kidney tumor in the UMCU medical records were reviewed and considered eligible if (1) the tumor was confirmed as sAML and (2) were never diagnosed with malignant renal tumors, TSC, or tuberculosis. (The latter were excluded because of possible misclassification in the hospital information system.) Pulmonary lymphangioleiomyomatosis [LAM] was not an exclusion criterion. Demographic and clinical information was extracted from the medical charts of eligible patients. The date of the first AML assessment at the UMCU was defined as the index date. Treatments, disease monitoring, and kidney function were measured from the index date to the date of death, date of the last follow-up at the UMCU, or date of data collection (April 2015), whichever came first (follow-up period). Pre-index information was collected from both the patient medical history taken at the time of the first AML assessment and, where applicable, from the patient's hospital records prior to the index date. All patients were seen, tested, and treated as part of the regular care at UMCU. The study was approved by the UMCU Institutional Review Board (study METC 14/412C).

Subgroups of interest were defined based on the size and number of AMLs at the index date, measured as part of the regular care at the UMCU. Patients with the largest AML at the index date of $\geq 3.5 \mathrm{~cm}$ formed the subgroup of patients with large AMLs, while patients with the largest AML at the index date of $<3.5 \mathrm{~cm}$ formed the subgroup of patients with small AMLs [17, 18]. Patients with small AMLs were further divided into patients with $\leq 5$ and $>5$ small AMLs, but stratification was not possible in the large AML subgroup where only two patients had $>5$ AMLs. The cutoffs for the size and number of AMLs correspond to the cutoffs between AML stages two and three in the AML staging criteria [17, 18] (Online Resource 1).

Treatments received included nephrectomies, embolizations, kidney transplants, and potential off-label mTORi. Disease monitoring was measured in terms of the use of diagnostic renal and non-renal scans (e.g., CT scans, ultrasounds), visits for sAML follow-up, and visits with specialists. Kidney function was based on glomerular filtration rate (eGFR) calculated from serum creatinine measurements. Characteristics of patients with sAML measured pre-index or at the index date are listed in Table 1. Hypertension was defined based on systolic and diastolic blood pressure measurements closest to the index date using NIH criteria [19]. Chronic kidney disease (CKD) stages were determined from eGFR levels [20, 21].

Rates of disease monitoring per patient per year (PPPY) were calculated as the number of scans/visits divided by the number of patient-years of follow-up and were compared between subgroups of interest using regressions with Poisson distribution. Mean eGFR levels were reported for several age groups such as 25 to 34 years and $\geq 65$ years by averaging the eGFR test values of all patients observed during the respective age groups. Mean eGFR levels across different ages were presented for comparison purposes alongside the corresponding mean eGFR levels across ages previously reported for the general Dutch population [22, 23] and the TSC population [18]. At each age, the mean eGFR levels from the general Dutch population $[22,23]$ were standardized to the sex distribution of the AML study sample at that age.

\section{Results}

\section{Patient characteristics}

The study sample included 53 patients, followed for a total of 184.6 patient-years. The large and small sAML subgroups included 26 and 27 patients, respectively. Only six patients 
Table 1 Patient characteristics at index date, overall, and stratified by size and number of renal AMLs

\begin{tabular}{|c|c|c|c|c|}
\hline & \multirow{4}{*}{$\begin{array}{l}\text { Patients with larg- } \\
\text { est } \mathrm{AML}^{\mathrm{a}} \text { at index } \\
\text { date } \geq 3.5 \mathrm{~cm} \\
(N=26)\end{array}$} & \multicolumn{3}{|c|}{ Patients with largest $\mathrm{AML}^{\mathrm{a}}$ at index date $<3.5 \mathrm{~cm}$} \\
\hline & & \multirow{3}{*}{$\begin{array}{l}\text { All patients } \\
(N=27)\end{array}$} & \multicolumn{2}{|c|}{ Stratification by the number of AMLs } \\
\hline & & & \multirow{2}{*}{$\begin{array}{l}\leq 5 \text { small AMLs } \\
-(N=21)\end{array}$} & \multirow{2}{*}{$\begin{array}{l}>5 \text { small AMLs } \\
(N=6)\end{array}$} \\
\hline & & & & \\
\hline Patient age (years), mean \pm SD [median] & $51.2 \pm 12.7[54.0]$ & $52.0 \pm 13.6[55.1]$ & $53.0 \pm 14.3[56.0]$ & $48.4 \pm 11.2[45.4]$ \\
\hline Female, $n(\%)$ & $22(84.6)$ & $23(85.2)$ & $17(81.0)$ & $6(100.0)$ \\
\hline Caucasian race, $n(\%)$ & $26(100)$ & $27(100)$ & $21(100)$ & $6(100)$ \\
\hline \multicolumn{5}{|l|}{ sAML stage $^{\mathrm{b}}, n(\%)$} \\
\hline 0 & $0(0.0)$ & $5(18.5)$ & $5(23.8)$ & $0(0.0)$ \\
\hline 1 & $0(0.0)$ & $14(51.9)$ & $13(61.9)$ & $1(16.7)$ \\
\hline 2 & $1(3.8)$ & $7(25.9)$ & $2(9.5)$ & $5(83.3)$ \\
\hline 3 & $19(73.1)$ & $1(3.7)$ & $1(4.8)$ & $0(0.0)$ \\
\hline 4 & $4(15.4)$ & $0(0.0)$ & $0(0.0)$ & $0(0.0)$ \\
\hline 5 & $1(3.8)$ & $0(0.0)$ & $0(0.0)$ & $0(0.0)$ \\
\hline 6 & $1(3.8)$ & $0(0.0)$ & $0(0.0)$ & $0(0.0)$ \\
\hline Bilateral renal AMLs, $n(\%)$ & $13(50.0)$ & $11(40.7)$ & $6(28.6)$ & $5(83.3)$ \\
\hline Prior sAML bleeding episodes, $n(\%)$ & $8(30.8)$ & $1(3.7)$ & $0(0.0)$ & $1(16.7)$ \\
\hline \multicolumn{5}{|l|}{ eGFR $\left(\mathrm{mL} / \mathrm{min} / 1.73 \mathrm{~m}^{2}\right)$ at index date $\mathrm{c}^{\mathrm{c}}, n(\%)$} \\
\hline$\geq 90$ (normal eGFR) & $6(23.1)$ & $10(37.0)$ & $6(28.6)$ & $4(66.7)$ \\
\hline 60-89 (CKD stage 2) & $14(53.8)$ & $11(40.7)$ & $9(42.9)$ & $2(33.3)$ \\
\hline 45-59 (CKD stage 3A) & $2(7.7)$ & $3(11.1)$ & $3(14.3)$ & $0(0.0)$ \\
\hline 30-44 (CKD stage 3B) & $0(0.0)$ & $0(0.0)$ & $0(0.0)$ & $0(0.0)$ \\
\hline 15-29 (CKD stage 4) & $0(0.0)$ & $0(0.0)$ & $0(0.0)$ & $0(0.0)$ \\
\hline Under 15 (CKD stage 5) & $0(0.0)$ & $0(0.0)$ & $0(0.0)$ & $0(0.0)$ \\
\hline Unknown & $4(15.4)$ & $3(11.1)$ & $3(14.3)$ & $0(0.0)$ \\
\hline Prior nephrectomy, $n(\%)$ & $2(7.7)$ & $4(14.8)$ & $3(14.3)$ & $1(16.7)$ \\
\hline Prior embolization, $n(\%)$ & $2(7.7)$ & $0(0.0)$ & $0(0.0)$ & $0(0.0)$ \\
\hline \multicolumn{5}{|l|}{ Hypertension at index date ${ }^{\mathrm{d}}, n(\%)$} \\
\hline Yes & $9(34.6)$ & $4(14.8)$ & $3(14.3)$ & $1(16.7)$ \\
\hline No & $4(15.4)$ & $5(18.5)$ & $3(14.3)$ & $2(33.3)$ \\
\hline Unknown & $13(50.0)$ & $18(66.7)$ & $15(71.4)$ & $3(50.0)$ \\
\hline LAM, $n(\%)$ & $4(15.4)$ & $3(11.1)$ & $2(9.5)$ & $1(16.7)$ \\
\hline AMLs in organs other than kidney or lung ${ }^{\mathrm{e}}, n(\%)$ & $2(7.7)$ & $0(0.0)$ & $0(0.0)$ & $0(0.0)$ \\
\hline History of pneumothorax, $n(\%)$ & $2(7.7)$ & $1(3.7)$ & $1(4.8)$ & $0(0.0)$ \\
\hline History of pulmonary nodule, $n(\%)$ & $2(7.7)$ & $2(7.4)$ & $2(9.5)$ & $0(0.0)$ \\
\hline $\begin{array}{l}\text { Duration post-index follow-up }{ }^{\mathrm{f}} \text { (years), mean } \pm \text { SD } \\
\text { [median] }\end{array}$ & $5.5 \pm 4.5[4.3]$ & $3.5 \pm 2.9[3.1]$ & $4.2 \pm 3.0[3.8]$ & $1.2 \pm 0.6[1.1]$ \\
\hline $\begin{array}{l}\text { Observation period prior to sAML diagnosis }{ }^{\mathrm{g}} \text { (years), } \\
\text { mean } \pm \text { SD [median] }\end{array}$ & $1.9 \pm 4.2[0.2]$ & $3.6 \pm 6.3[0.6]$ & $4.8 \pm 7.2[0.6]$ & $0.8 \pm 0.5[1.0]$ \\
\hline
\end{tabular}

$s A M L$, sporadic angiomyolipoma; $S D$, standard deviation; $L A M$, lymphangioleiomyomatosis; $e G F R$, estimated glomerular filtration rate

${ }^{a}$ As reported in the chart; the subgroup with $\leq 5$ small AMLs includes one patient without any renal AMLs at index date (the patient had nephrectomy pre-index)

${ }^{\mathrm{b}}$ As reported in the chart; please see Online Resource 1 for the renal AML staging criteria used in this study

${ }^{\mathrm{c}}$ Reported as part of the sAML assessment for almost all patients

${ }^{\mathrm{d}}$ Defined as systolic pressure $\geq 140 \mathrm{mmHg}$ or diastolic pressure $\geq 90 \mathrm{mmHg}$ (source: NIH MedlinePlus, http://www.nlm.nih.gov/medlinep lus/magazine/issues/winter10/articles/winter10pg10a.html, accessed August 13, 2015). For patients with multiple blood pressure measurements within this period, hypertension status is based on the measurement closest to the index date within 90 days before or after the index date

${ }^{\mathrm{e}}$ One patient had AMLs in the pancreas; another patient had AMLs in the liver

${ }_{\mathrm{f}}^{\mathrm{f}}$ From index date to date of the last recorded visit or date of data collection (April 2015), whichever came first

${ }^{\mathrm{g}}$ From first recorded visit to sAML diagnosis 
in the small sAML subgroup had $>5$ AMLs, but they accounted for $75 \%$ of all patients with $>5$ AMLs (Table 1 , Fig. 1). The largest AML observed in the study sample was $18 \mathrm{~cm}$ (Fig. 1).

Median age at index date ranged from 48 to 53 years across subgroups (Table 1). Patients with large AMLs had the highest frequency for the history of sAML bleeding (31\%) and hypertension (35\%), but the lowest rate of preindex nephrectomy (8\%). No patient had CKD stage $\geq 3 \mathrm{~B}$ at the index date. Bilateral renal AMLs were found in $83 \%$ of patients with $>5$ small AMLs and $50 \%$ of patients with large AMLs. LAM was observed in $10-17 \%$ of the patients, depending on the group. AML in other organs was reported in two patients, both in the large AML subgroup.

\section{Treatments}

In the post-index period, six (11\%) patients underwent a nephrectomy and two (4\%) patients underwent an embolization. (One patient underwent both interventions.) These interventions appeared to be more common among patients with large AMLs than patients with small AMLs (19 vs. $4 \%$ and 8 vs. $0 \%$, respectively; Fig. 2), and there were no kidney transplants. Off-label mTORi were received by five (9\%) of patients (four [15\%] and one [17\%] patients with large AMLs and $>5$ small AMLs, respectively), with three patients experiencing a decrease in AML size post-mTORi initiation and two patients experiencing no change in AML size (Online Resource 2).

\section{Disease monitoring}

Renal scan monitoring was highest for patients with $>5$ small AMLs and lowest for patients with $\leq 5$ small AMLs

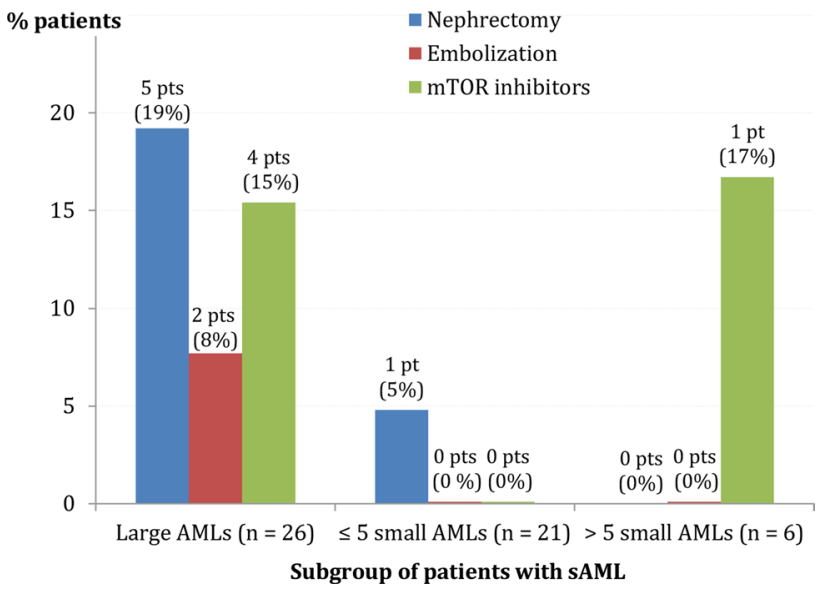

Fig. 2 Treatments received during the follow-up period. $s A M L$, sporadic angiomyolipoma; $m$ TOR inhibitor, inhibitor of mammalian target of rapamycin; $p t$, patient

(Fig. 3a). Patients with large AMLs had significantly higher rates of renal scans compared with patients with small AMLs, but lower rates than the subgroup of patients with $>5$ small AMLs. Patients with $>5$ small AMLs used significantly more renal scans than patients with $\leq 5$ small AMLs, with the exception of renal ultrasounds. Non-renal scan use, mainly CT scans and MRIs, was similar in patients with large and small AMLs, but significantly higher in patients with $>5$ versus $\leq 5$ small AMLs.

Frequency of sAML follow-up visits post-index was also highest for patients with $>5$ small AMLs and lowest for patients with $\leq 5$ small AMLs (Fig. 3b). There was a nonstatistically significant trend toward higher rates of sAML follow-up visits among patients with large AMLs (1.07 visits PPPY) compared to patients with small AMLs (0.94 visits
Fig. 1 Number and size of AMLs at index date among the 53 patients in the sAML study sample

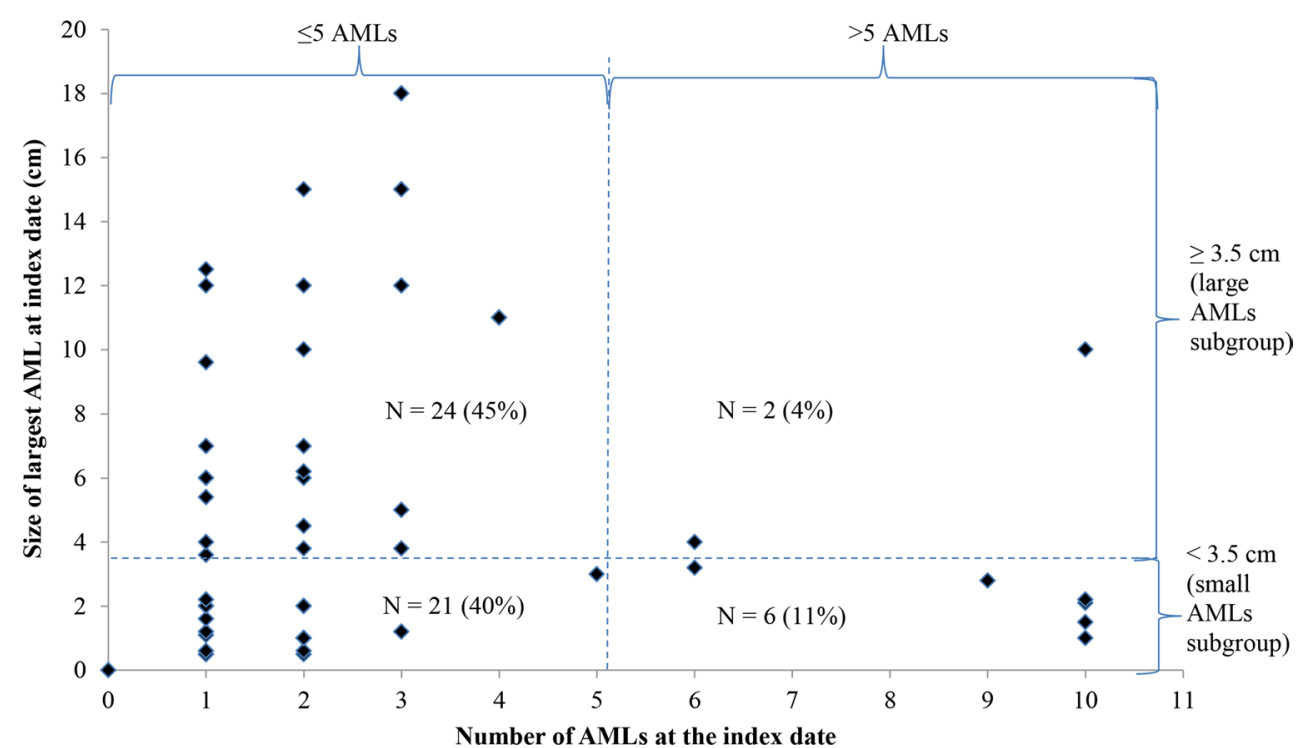




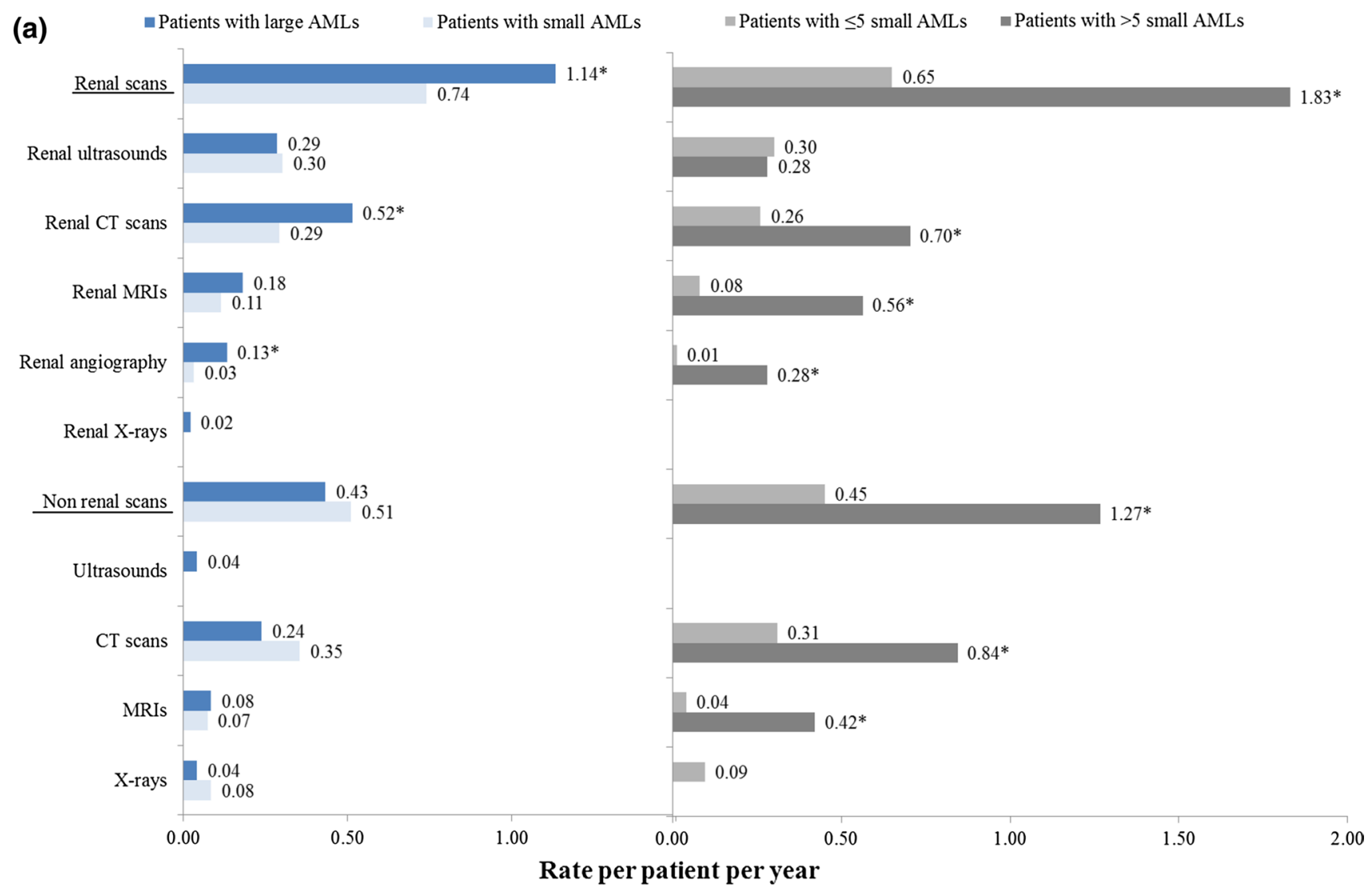

(b)

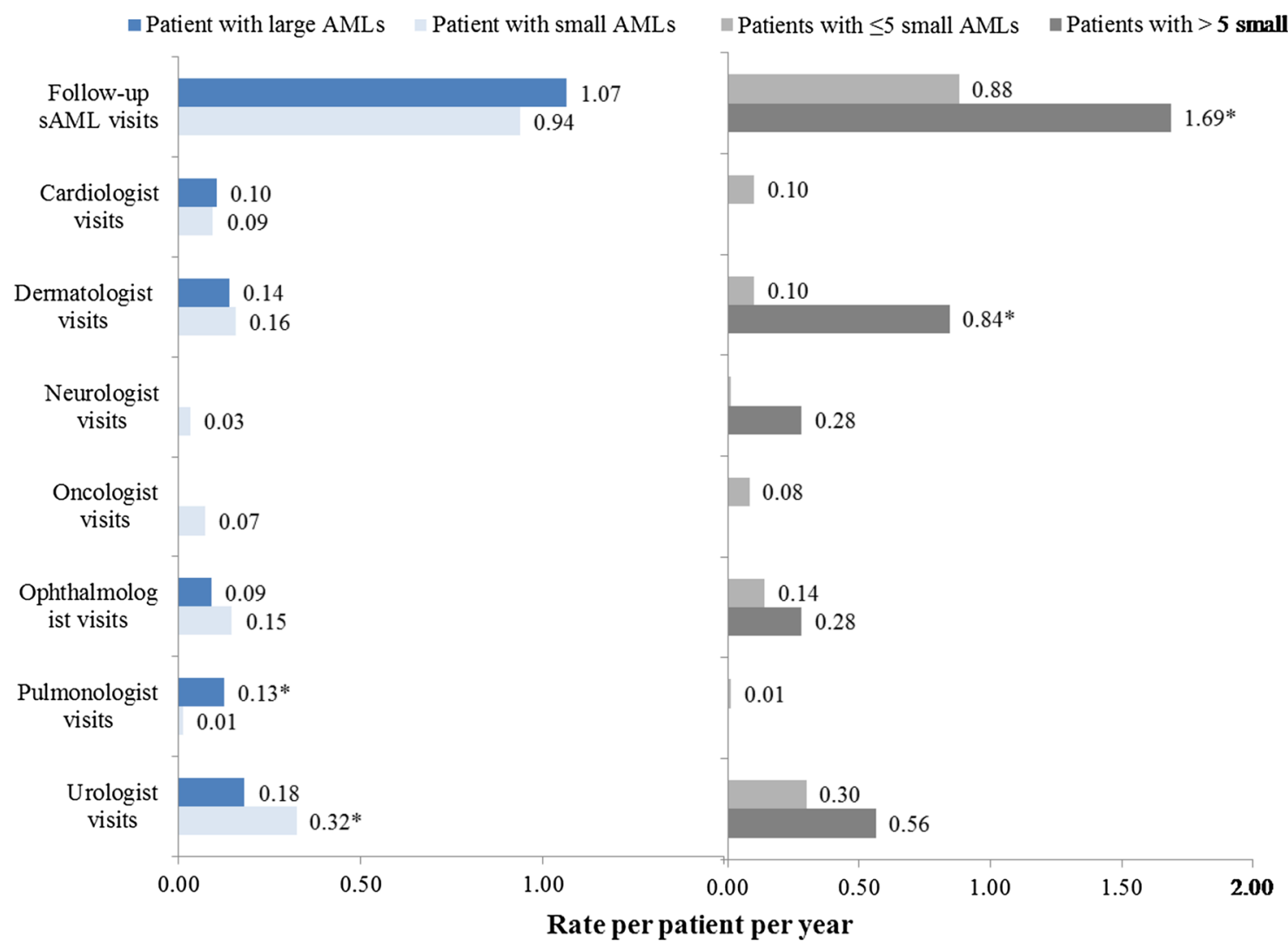

Fig. 3 Disease monitoring in the follow-up period. Panel a Scans. Panel b Visits. *Rates statistically different between groups $(P<0.05)$ 
PPPY, $\mathrm{P}=0.342$ ). As compared to patients with $\leq 5$ small AMLs, patients with $>5$ small AMLs had significantly higher rates of visits to pulmonologists $(0.13$ vs. 0.01 visits PPPY, $P=0.016)$ and dermatologist (0.84 vs. 0.10 visits PPPY, $P<0.001)$ and lower rates of visits to urologist $(0.18$ vs. 0.32 visits PPPY, $P=0.029)$.

\section{Renal function}

As compared to patients with small AMLs, the mean eGFR of patients from the sAML study sample with large AMLs was lower at young ages ( 87.0 vs. $108.1 \mathrm{~mL} / \mathrm{min} / 1.73 \mathrm{~m}^{2} /$ year at the age of 25-34 years; 81.1 vs. 100.8 at 35-44 years; 77.0 vs. 92.8 at $45-54$ years) and higher at older ages (77.4 vs. 60.8 at $\geq 65$ years; Table 2 ). Patients with sAML developed CKD stage two at a younger age on average compared to patients in the general Dutch population [23] (age 42 vs. 55 years), but later than the patients with TSC (age 42 vs. 35 years; Fig. 4) [18]. Patients on average had 5.9 eGFR measurements over the follow-up period. Among patients with 10 or more eGFR measurements $(N=6)$, the trends were mixed (Online Resource 3).

\section{Discussion}

This study investigated the natural history and treatment of 53 patients with sAML followed in a large tertiary center in the Netherlands, overall and stratified by the size and number of AMLs. Large AMLs were common in this population, posed a high burden on the patient in the form of comorbidities and invasive interventions and required closer monitoring than small AMLs. The $25 \%$ of the patients with small AMLs who had > 5 small AMLs had the highest rates in the sAML population for renal scans and follow-up sAML visits. The results from the study also support the hypothesis that AMLs impact kidney function, as indicated by the earlier onset of renal impairment in both patients with sAML and TSC as compared to the general population.
Table 2 eGFR by age group and size of AMLs

Fig. 4 eGFR by age among patients in the sAML study sample ${ }^{\mathrm{a}}$, patients with $\mathrm{TSC}^{\mathrm{b}}$, and the general Dutch population $^{\mathrm{c}}$. $e G F R$, estimated glomerular filtration rate; $s A M L$, sporadic angiomyolipoma; $T S C$, tuberous sclerosis complex. ${ }^{\mathrm{a}} \mathrm{A}$ patient may contribute multiple times to a given age category and to multiple age categories. ${ }^{\mathrm{b}}$ Data points by age extracted from Vekeman et al. JME 2015. ${ }^{c}$ Data points by age group extracted from Wetzels JFM et al. Kidney International 2007 standardized to the sex distribution in the sAML study sample

\begin{tabular}{|c|c|c|c|c|c|}
\hline & \multicolumn{5}{|c|}{ Mean eGFR $\left(\mathrm{mL} / \mathrm{min} / 1.73 \mathrm{~m}^{2}\right)$} \\
\hline & Age $25-34^{\mathrm{a}}$ & Age $35-44^{\mathrm{a}}$ & Age $45-54^{\mathrm{a}}$ & Age $55-64^{\mathrm{a}}$ & Age $\geq 65^{\mathrm{a}}$ \\
\hline All patients with sAML & $98.9(6 \mathrm{pts})$ & 86.8 (14 pts) & 80.3 (13 pts) & 75.6 (24 pts) & $67.3(14 \mathrm{pts})$ \\
\hline Large AMLs subgroup & 87.0 (3 pts) & 81.1 (8 pts) & 77.0 (7 pts) & 74.0 (14 pts) & 77.4 (8 pts) \\
\hline Small AMLs subgroup ${ }^{b}$ & 108.1 (3 pts) & 100.8 (6 pts) & 92.8 (6 pts) & $78.2(10 \mathrm{pts})$ & 60.8 (6 pts) \\
\hline
\end{tabular}

$e G F R$, estimated glomerular filtration rate; $A M L$, angiomyolipoma; pts, patients

${ }^{a}$ One patient may have contributed multiple times to a given age category and to multiple age categories

${ }^{b}$ Age group stratification was not possible for the subgroups of patients with $\leq 5$ small AMLs and $>5$ small AMLs due to the small number of patients

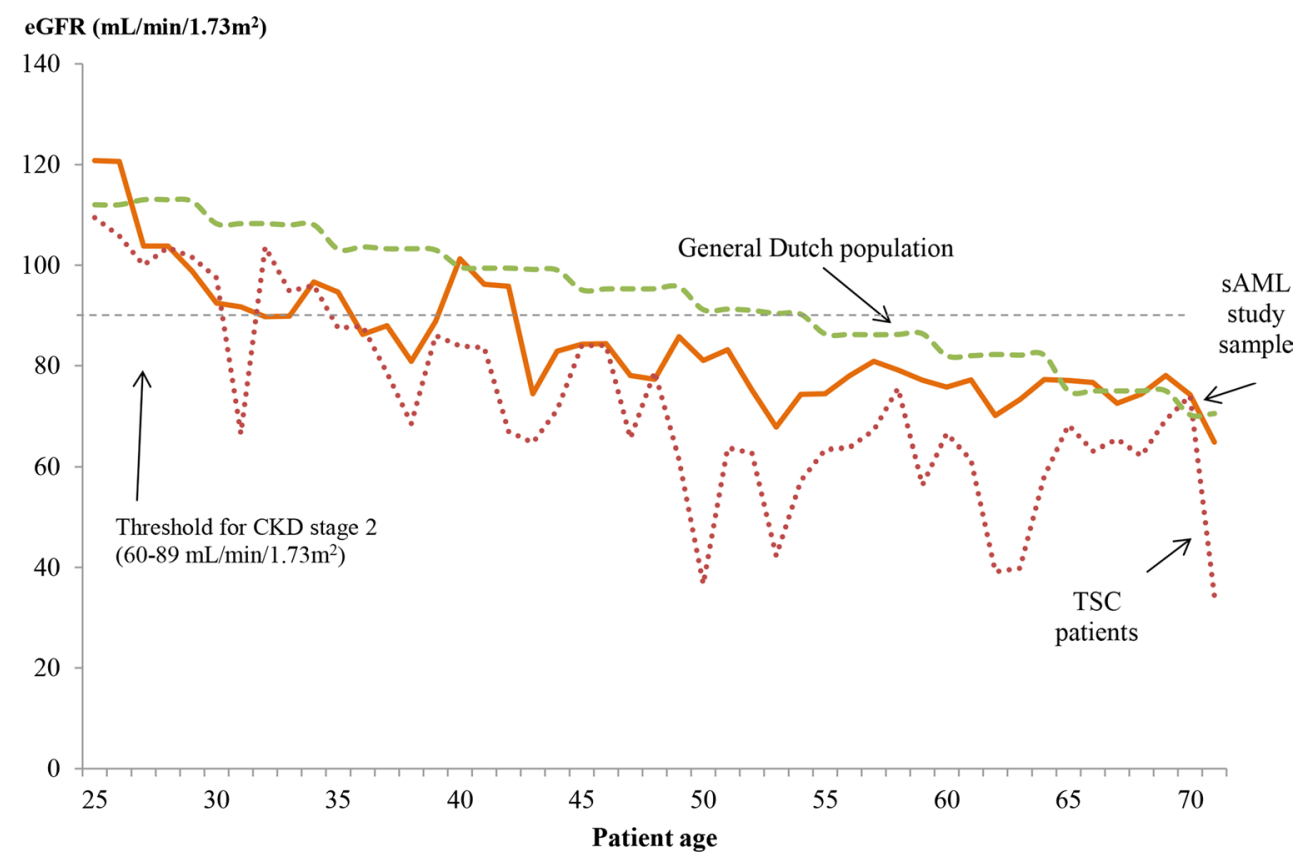


The literature on SAML is scarce, with most previous studies relying on small case series or case series aggregated data, often without distinguishing between patients with sAML and patients with TSC-AML $[3,6,8,11]$. With a median age of 54 years and a predominance of females, patients in the current study have a similar age and sex distribution to patients with AMLs in previous studies $[6,11$, $24,25]$. However, advanced sAML may have been more common at the index date in the current study as compared to other studies: $43 \%$ patients had tumors $>4 \mathrm{~cm}$ [data not shown] versus $14-22 \%$ in other studies $[9,25], 36 \%$ had a single AML versus $77-80 \%[11,25]$ and $45 \%$ had bilateral AML versus $5-16 \%[9,11]$. Consistent with this finding, more patients in the current study underwent a nephrectomy or embolization during their lifetime (26\%) than patients in other contemporary studies (range 5.6-13\%) [7, 11]. These intervention rates are nevertheless lower than the rates observed in older studies [3,10], reflecting a possible shift in sAML disease management, where active surveillance is considered a safer practice, and better imaging techniques reduce the unnecessary nephrectomy related to misinterpretation of the AML as a renal carcinoma [11].

As expected based on current guidelines [14], nephrectomy and embolization were performed mainly on patients with large AMLs. Other studies have also showed that $86-100 \%$ of all interventions were in patients with large AMLs [6,9]. Interestingly, more patients with sAML underwent nephrectomy than embolization than was previously reported for TSC-AML $[17,26]$. This may be due to the aforementioned possible shift in clinical practice if the sAML and TSC-AML patients from the two studies reflected different treatment eras, but it is also possible that nephrectomies may be considered more risky interventions in TSCAML. Five patients $(9.4 \%)$ in the current sample received treatment with mTORi, including four with large AMLs (range: 7-18 cm) and four with CKD at the time of treatment initiation (eGFR range: 65-85). Interestingly, all three patients with CKD who had eGFR measurements before and after mTORi initiation had an improvement in eGFR after mTORi initiation (range: $8-15 \%$ eGFR increase; data not shown). These results suggest that mTORi could play a role in these patients, as previously hypothesized based on the presence of inappropriately regulated mTOR pathway in sAML [16].

The current study also found that rates of renal scans and rates of follow-up visits for sAML were higher among patients with large AMLs and patients with $>5$ small AMLs. The finding that patients with large AMLs are monitored closely is in line with current recommendations for patients known to be at an increased risk of complications such as spontaneous hemorrhage or flank pain [26, 27]. While patients with small AMLs were not traditionally considered to be high-risk patients, recent studies showing that AMLs may grow faster when multiple tumors are present [28] may have changed the risk assessment for these patients. However, it is also possible that sAML differential diagnosis is more difficult in patients with small AMLs, thus requiring more testing to potentially rule out TSC or malignant neoplasms. The study finding that patients with $>5$ small AMLs also had high rates of non-renal scans and visits to dermatologist and ophthalmologists suggests that at least some of these patients undergo testing for TSC.

This study has a number of limitations. First, results cannot be generalized to the entire sAML population and reflect the patients followed in specialized tertiary centers in the Netherlands. Second, differences in renal function between patients at different age groups should not be interpreted as rates of decline by age in renal function because different patients may have contributed to different age groups. Third, part of the kidney function decline in patients with nephrectomy prior to the index date may be related to the prior nephrectomy. Given that patients with small AMLs had higher frequency of prior nephrectomies, the eGFR gap observed between the large and small AML subgroups may have been underestimated at younger ages and overestimated at older ages. Fourth, information on treatment received outside the UMCU was limited to the physician notes and may be subject to missing information. However, all major interventions and key events that occurred outside the UMCU were likely captured in the patients' medical history. Fifth, imaging tests have become in recent years more sensitive to detect small AMLs. This is consistent with the study finding that patients with $>5$ small AMLs had shorter follow-up (i.e., were diagnosed as such more recently) than patients with $\leq 5$ small AMLs. The true proportion of patients with $>5$ small AMLs may be underestimated.

In conclusion, large AMLs are common among patients with sAML and pose a high burden on the patient in the form of comorbidities and invasive interventions. As compared to patients with small AMLs, patients with large AMLs were more likely to have bilateral renal AMLs, LAM, hypertension, AML bleeding at the study onset; appeared to be at an increased risk of undergoing a nephrectomy, embolization, and having early onset of kidney impairment; and had higher rates of renal scans. While the subgroup of patients with $>5$ small AMLs included only six patients, most of these patients had bilateral renal AMLs and some patients also had a history of bleeding and nephrectomy, suggesting this subgroup may also pose a high comorbidity and interventional burden. Furthermore, these patients had high rates of renal scans and follow-up sAML visits as compared to patients with $\leq 5$ small AMLs. Due to the potential of sAMLs to grow over time and the detrimental and costly long-term clinical outcomes for patients with large and/or multiple AMLs, there is an urgent need for therapies targeting a possible mTOR pathway in AML and for interventions that can 
prevent or delay the growth of AMLs without impairing the kidney.

Acknowledgements The authors would like to thank Pim Kuizenga, an employee of Wellmera AG, for his comments on the manuscript.

Author's contributions JLHRB is an employee of the University Medical Center Utrecht, which received funding from Novartis for the collection of the data used in this study. BAZ, currently retired, was formerly an employee of the University Medical Center Utrecht, which received funding from Novartis for the collection of the data used in this study. MN is an employee of Novartis Pharmaceuticals Corporation. MM (currently EMD Serono, Inc.) was an employee of Novartis Pharmaceuticals Corporation at the time the study was conducted. MD and RI-I are employees of Analysis Group, Inc., which has received consultancy fees from Novartis for this study. At the time the study was conducted, PK, JF, and FV were employees of Analysis Group, Inc., which has received consultancy fees from Novartis for this study.

Funding Funding for this research was provided by Novartis Pharmaceuticals Corporation. Analysis Group, Inc., has received consultancy fees from Novartis to assist in designing of the study and performing of the analyses. University Medical Center Utrecht was subcontracted by the Analysis Group, Inc., for the data collection.

Open Access This article is distributed under the terms of the Creative Commons Attribution 4.0 International License (http://creativecomm ons.org/licenses/by/4.0/), which permits unrestricted use, distribution, and reproduction in any medium, provided you give appropriate credit to the original author(s) and the source, provide a link to the Creative Commons license, and indicate if changes were made.

\section{References}

1. Skolarus TA, Serrano MF, Berger DA et al (2008) The distribution of histological subtypes of renal tumors by decade of life using the 2004 WHO classification. J Urol 179(2):439-443. https://doi. org/10.1016/j.juro.2007.09.076 (discussion 443-4)

2. Fittschen A, Wendlik I, Oeztuerk S et al (2014) Prevalence of sporadic renal angiomyolipoma: a retrospective analysis of 61,389 in- and out-patients. Abdom Imaging 39(5):1009-1013. https:// doi.org/10.1007/s00261-014-0129-6

3. Nelson CP, Sanda MG (2002) Contemporary diagnosis and management of renal angiomyolipoma. J Urol 168(4 Pt 1):1315-1325. https://doi.org/10.1097/01.ju.0000028200.86216.b2

4. Rabenou RA, Charles HW (2015) Differentiation of sporadic versus tuberous sclerosis complex-associated angiomyolipoma. AJR Am J Roentgenol 205(2):292-301. https://doi.org/10.2214 /AJR.14.14255

5. Coombs EJ (2013) Role of mTOR inhibition in the treatment of patients with renal angiomyolipomas. J Am Assoc Nurse Pract 25(11):588-596. https://doi.org/10.1002/2327-6924.12081

6. Seyam RM, Bissada NK, Kattan SA et al (2008) Changing trends in presentation, diagnosis and management of renal angiomyolipoma: comparison of sporadic and tuberous sclerosis complex-associated forms. Urology 72(5):1077-1082. https://doi. org/10.1016/j.urology.2008.07.049

7. Ouzaid I, Autorino R, Fatica R et al (2014) Active surveillance for renal angiomyolipoma: outcomes and factors predictive of delayed intervention. BJU Int 114(3):412-417. https://doi.org/10.1111/ bju.12604
8. Sooriakumaran P, Gibbs P, Coughlin G et al (2010) Angiomyolipomata: challenges, solutions, and future prospects based on over 100 cases treated. BJU Int 105(1):101-106. https://doi. org/10.1111/j.1464-410X.2009.08649.x

9. Al-Thani H, El-Menyar A, Al-Sulaiti M et al (2014) Clinical presentation, management, and outcome of patients with incidental renal angiomyolipoma in Qatar. Oman Med J 29(6):419-424. http s://doi.org/10.5001/omj.2014.112

10. Mues AC, Palacios JM, Haramis G et al (2010) Contemporary experience in the management of angiomyolipoma. J Endourol 24(11):1883-1886. https://doi.org/10.1089/end.2010.0223

11. Bhatt JR, Richard PO, Kim NS et al (2016) Natural history of renal angiomyolipoma (AML): most patients with large AMLs $>4 \mathrm{~cm}$ can be offered active surveillance as an initial management strategy. Eur Urol 70(1):85-90. https://doi. org/10.1016/j.eururo.2016.01.048

12. Albi G, del Campo L, Tagarro D (2002) Wünderlich's syndrome: causes, diagnosis and radiological management. Clin Radiol 57(9):840-845. https://doi.org/10.1016/S0899-7071 (03)00058-5

13. Zhang JQ, Fielding JR, Zou KH (2002) Etiology of spontaneous perirenal hemorrhage: a meta-analysis. J Urol 167(4):1593-1596. https://doi.org/10.1016/S0022-5347(05)65160-9

14. Ljungberg B, Bensalah K, Canfield $\mathrm{S}$ et al (2015) EAU guidelines on renal cell carcinoma: 2014 update. Eur Urol 67(5):913-924. https://doi.org/10.1016/j.eururo.2015.01.005

15. Bissler JJ, Kingswood JC (2004) Renal angiomyolipomata. Kidney Int 66(3):924-934. https://doi.org/10.1111/j.1523-1755.2004 $.00838 . \mathrm{x}$

16. Shitara K, Yatabe Y, Mizota A, Sano T, Nimura Y, Muro K (2011) Dramatic tumor response to everolimus for malignant epithelioid angiomyolipoma. Jpn J Clin Oncol 41(6):814-816. https://doi. org/10.1093/jjco/hyr035

17. Eijkemans MJC, van der Wal W, Reijnders LJ et al (2015) Longterm follow-up assessing renal angiomyolipoma treatment patterns, morbidity, and mortality: an observational study in tuberous sclerosis complex patients in the Netherlands. Am J Kidney Dis 66(4):638-645. https://doi.org/10.1053/j.ajkd.2015.05.016

18. Vekeman F, Magestro M, Karner P et al (2015) Kidney involvement in tuberous sclerosis complex: the impact on healthcare resource use and costs. J Med Econ 18(12):1060-1070. https:// doi.org/10.3111/13696998.2015.1075995

19. Chobanian AV, Bakris GL, Black HR et al (2003) The seventh report of the joint national committee on prevention, detection, evaluation, and treatment of high blood pressure: the JNC 7 report. JAMA 289(19):2560-2572. https://doi.org/10.1001/jama .289 .19 .2560

20. Eckardt K-U, Berns JS, Rocco MV, Kasiske BL (2009) Definition and classification of CKD: the debate should be about patient prognosis - a position statement from KDOQI and KDIGO. Am J Kidney Dis 53(6):915-920. https://doi.org/10.1053/j.ajkd.2009 .04 .001

21. Levey AS, Stevens LA, Schmid CH et al (2009) A new equation to estimate glomerular filtration rate. Ann Intern Med 150(9):604 612. https://doi.org/10.7326/0003-4819-150-9-200905050-00006

22. Van den Brand JAJG, van Boekel GAJ, Willems HL, Kiemeney LALM, den Heijer M, Wetzels JFM (2011) Introduction of the CKD-EPI equation to estimate glomerular filtration rate in a Caucasian population. Nephrol Dial Transplant 26(10):3176-3181. https://doi.org/10.1093/ndt/gfr003

23. Wetzels JFM, Kiemeney LALM, Swinkels DW, Willems HL, den Heijer M (2007) Age- and gender-specific reference values of estimated GFR in Caucasians: the Nijmegen biomedical study. Kidney Int 72(5):632-637. https://doi.org/10.1038/sj.ki.5002374

24. Boorjian SA, Frank I, Inman B et al (2007) The role of partial nephrectomy for the management of sporadic renal 
angiomyolipoma. Urology 70(6):1064-1068. https://doi. org/10.1016/j.urology.2007.07.045

25. Ryu JH, Hartman TE, Torres VE, Decker PA (2012) Frequency of undiagnosed cystic lung disease in patients with sporadic renal angiomyolipomas. Chest 141(1):163-168. https://doi.org/10.1378 /chest.11-0669

26. Koo KC, Kim WT, Ham WS, Lee JS, Ju HJ, Choi YD (2010) Trends of presentation and clinical outcome of treated renal angiomyolipoma. Yonsei Med J 51(5):728-734. https://doi.org/10.3349 /ymj.2010.51.5.728
27. Sivalingam S, Nakada SY (2013) Contemporary minimally invasive treatment options for renal angiomyolipomas. Curr Urol Rep 14(2):147-153. https://doi.org/10.1007/s11934-013-0311-3

28. Maclean DFW, Sultana R, Radwan R, McKnight L, Khastgir $\mathrm{J}$ (2014) Is the follow-up of small renal angiomyolipomas a necessary precaution? Clin Radiol 69(8):822-826. https://doi. org/10.1016/j.crad.2014.03.016 